\title{
Tolerancing and Sheet Bending in Small Batch Part Manufacturing
}

\author{
L. J. de Vin, A. H. Streppel, H. J. J. Kals (1), \\ Laboratory of Production and Design Engineering, University of Twente, The Netherlands \\ Received on January 12,1994
}

\begin{abstract}
Tolerances indicate geometrical limits between which a component is expected to perform its function adequately. They are used for instance for set-up selection in process planning and for inspection. Tolerances must be accounted for in sequencing and positioning procedures for bending of sheet metal parts. In bending, the shape of a part changes not only locally, but globally as well. Therefore, sheet metal part manufacturing presents some specific problems as regards reasoning about tolerances. The paper focuses on the interpretation and conversion of tolerances as part of a sequencing procedure for bending to be used in an integrated CAPP system.
\end{abstract}

Keywords: Tolerancing, Sheetmetal, CAPP

\section{INTRODUCTION}

\subsection{Tolerances}

Tolerances in technical drawings or CAD-models indicate limits between which a component is expected to perform its function adequately. In process planning, tolerance prescriptions are used in the procedures for the selection of machining methods and equipment. In inspection, they serve as reference for the measurement of the actual accuracy of the component. The interpretation of tolerances must be unambiguous and identical for the three above mentioned purposes. In practice however, these requirements are not always met, even when components are toleranced according to standards such as ISO 1101.

In bending of sheet metal parts, tolerances are, together with the handling and collision aspects, the basis for sequencing and positioning [21, 22]. However, the theory of tolerancing is merely directed to applications for prismatic and rotational parts and for assemblies of these parts $[1,3,15,24]$. For instance, the conversion of tolerances between subsequent product states in bending is not supported.

This paper addresses some important aspects of the interpretation and conversion of tolerances for sheet metal components.

\subsection{The PART-S system}

PART-S (Planning of Activities, Resources and Technology Sheet metal) is a prototype of a CAPP system for sheet metal components, presently under development at the Laboratory of Production and Design Engineering [20, 21]. The products considered represent 2D and 3D components with straight bends and/or welds, and with a sheet thickness between 0.5 and $5 \mathrm{~mm}$. The main operations considered are punching, nibbling, laser cutting, laser welding, and air bending on CNC press brakes. A typical application area is a job shop environment with NC and CNC equipment. PART-S covers the whole range of decision making from the interpretation of CAD product models (through recognition of manufacturing form features) to the generation of NC programs. It is an extension of a previously developed CAPP system for prismatic parts, called PART [8]. PART forms the basis of a commercially available CAPP system. PART and PART-S are configurable with respect to company specific planning scenarios $[9,21]$. Integration with production planning is achieved by performing the workloading task for the flat pattern operations within the process planning cycle $[12,19,23]$. In this way, the relations between nesting, workloading and tool management are addressed. A schematic representation of the PART-S system, showing a decomposition into functional modules, is given in Figure 1. The interpretation and conversion of tolerances for the determination of bending set-ups, which is the main topic discussed in this paper, is located in the HPCC module.

\subsection{The generation of bending sequences}

The determination of the bending sequence can be divided into five subfunctions. Following the 'reverse bending sequence' approach to the sequencing problem, the final product is the starting point and the bends are flattened one by one until the flat product state is reached $[7,17,21]$.

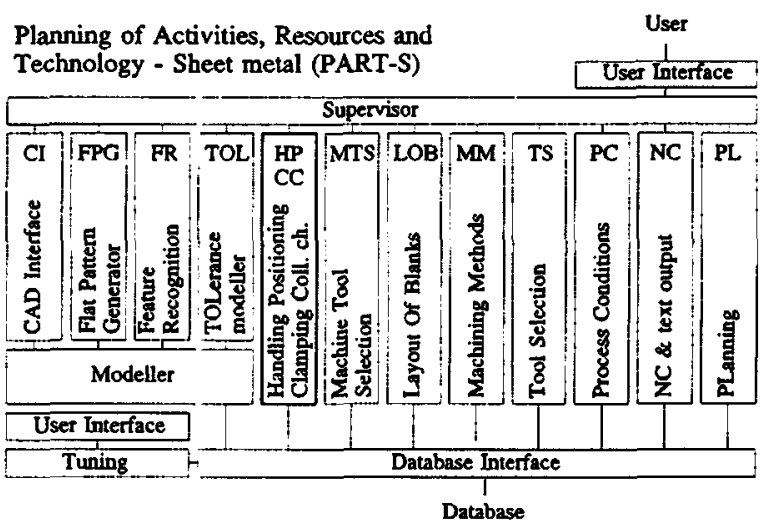

Figure 1: Schematic representation of the PART-S system

A reduction of the search space: A reduction of the search space is possible by the elimination of non-feasible solutions (e.g. sub-sequences which will inevitably lead to collisions).

A reduction of the tolerance scheme: In a product model, usually many toleranced dimensions are present. Tolerances which are not influenced by any bending operation can be disregarded during the generation of bending sequences.

The generation of alternative preceding set-ups: In a given product state (final or intermediate), usually different preceding states are possible, each corresponding with a different set-up in manufacturing. A model of such a previous state can be created by unfolding a bend in the CAD model, followed by the conversion of tolerances. When any tolerance prescription cannot be satisfied, the corresponding set-up is removed from the set of alternative set-ups. This procedure is followed for each feasible bend in the (intermediate) product model. In this way, for a given product state, alternative preceding states are determined.

The selection of a solution from the set of alternatives: Each alternative preceding state is evaluated using rules concerning accuracy, handling aspects and tooling. Usually, the set of rules consists of numerical and heuristic rules [14]. From the set of alternatives, the most promising solution is selected. This is the solution which has the highest potential in terms of leading to a fair overall solution.

A collision check: A collision check is required since general rules cannot cover all possible situations. The check is performed in simulation mode and proves whether parts of the product interfere with other parts of the product or with the manufacturing equipment [10].

\section{THE INTERPRETATION AND REPRESENTATION OF TOLERANCES}

\subsection{Tolerancing}

Basically, tolerances can be divided into two types: (i) Conventional dimensional ( \pm ) tolerances and (ii) Geometrical tolerances.

Although the use of tolerances of the first type is gradually being abandoned by many designers [4], these tolerances 
are still widely used. Their interpretation is ambiguous when components deviate from nominal geometry. Therefore, a convention for the interpretation of conventional tolerances is required [22]. The convention proposed below is based on the replacement of the conventional tolerances by position tolerances in such a way, that the designers' intent is not violated. a)

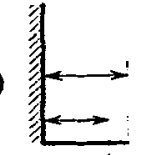

b)

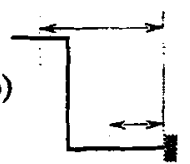

Main face as

reference face

Side face as

reference face
Figure 2: Reference faces and toleranced objects for sheetmetal parts

Some cross sections of sheet metal components are shown in Figure 2. A reference face and a toleranced face are defined for each conventional tolerance. The expression 'reference face' is used here instead of 'datum face', since a face doesn't usually serve as datum, but only serves as a reference to define a datum p/ane [25]. Figure $2 a$ shows tolerances between flat pattern form features (such as holes, notches, side faces) and a 'main' face. In that case, the main face is appointed reference face. Otherwise, the tolerance would imply an unintended additional angularity tolerance. A situation in which the face which is most frequently used to define tolerances is selected as the reference face is shown in Figure $2 \mathrm{~b}$. In this case, it is assumed that the side face is exactly perpendicular with respect to its 'main' face. This implies that the side face is used to establish a secon. dary datum, perpendicular to the primary datum attached to the main face.

Apart from position tolerances, the geometrical tolerances most commonly used in sheet metal are parallelness, perpendicularity and angularity tolerances. These tolerances only influence the required angular accuracy of bending operations. They can for instance serve as a criterium to select equipment. Position errors for instance, leave this type of tolerances invariant. Figure 3 shows a component with a paralleiness tolerance. The toleranced face must lie within the tolerance zone. When a position tolerance is used instead of a parallelness tolerance, the distance between the tolerance zone and the datum plane is prescribed. In principle, the datum plane is infinite, whereas the tolerance zones are also infinite in the directions parallel to the datum plane.
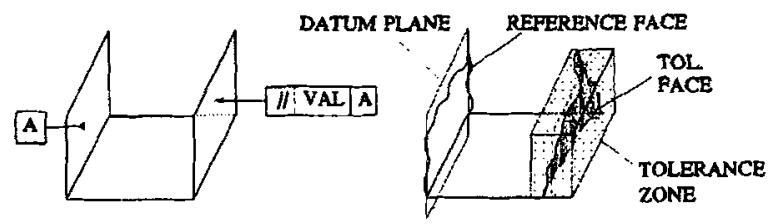

Figure 3: The tolerance zone of a paralleiness tolerance

2.2 The determination and representation of tolerances A problem in present tolerancing practice is that not always the correct type of tolerance is used. In addition, tolerance prescriptions are often stricter than necessary. This may lead to unnecessarily high manufacturing costs. In other cases, a component may not be manufacturable at all. In some cases, components which have not been manufactured according to the tolerance prescriptions are not rejected by the customer. This can lead to a certain casualness in the use of tolerances in the workshop. Since this is a very undesirable situation, it is important to specify tolerances correctly in the design stage.

The problem of non correctly specified tolerances has been recognized by many authors. Research in the field of Technologically and Technically Related Surfaces (TTRS's) is directed towards a systematic analysis of the relations between faces and admissible mutual degrees of freedom with respect to position and orientation $[4,5]$. Other approaches towards the tolerancing problem are tolerance analysis with the aid of Monte Carlo simulations and tolerance synthesis with the use of genetic algorithms $[11,13]$. Also the compatibility of tolerance schemes is addressed [16]. Many modellers do not allow tolerance modelling other than the addition of textual information. Also, tolerance transfer between modellers is in general not supported. However, in the design stage, tolerances can be attached to faces of features as additional information [6]. This is a representation method which has also proved to be suitable for process planning applications [2]. An example is shown in Figure 4.

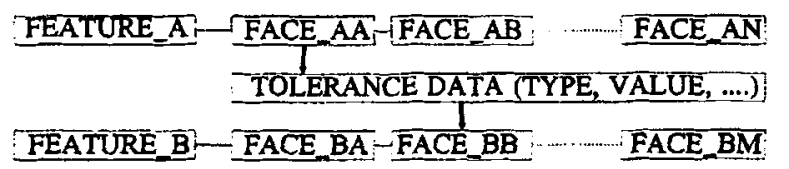

Figure 4: Tolerances can be represented as relations between faces of features (Feature $A$ serves as reference)

\section{AIR BENDING}

\subsection{The air bending process}

The main process stages in air bending are presented in Figure 5. A component (in this case a flat blank) is positioned against the finger stops and clamped between punch and die. Next, the punch is lowered to a precalculated position, after which it is retracted. The punch displacement, the tool geometry, the sheet thickness and the material behaviour determine the resulting bend angle after unloading.

\subsection{Errors in air bending}

Presently, three types of bending errors are distinguished in PART-S: (i) Angularity errors, (ii) positioning errors and (iii) errors in length. The errors of these three types will in general not compensate each other. The positioning errors result from the positioning of the part on the press brake. The error in length and the error in angle are both caused by the bending process [18]. Figure 6 shows an example of a bending error. The product radius is $0.2 \mathrm{~mm}$ larger than intended. As a consequence, each straight section is 0.16 $\mathrm{mm}$ shorter $(1 / 2 * \pi / 2 * 0.2 \mathrm{~mm})$. This results in a one-sided error in length of $0.04 \mathrm{~mm}$. The errors of both sections have equal signs (in this example, positive signs). This contrary to a positioning error, which is positive for one section and negative for the other.
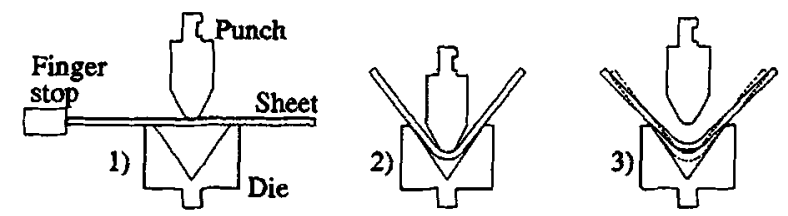

Figure 5: The main stages in air bending

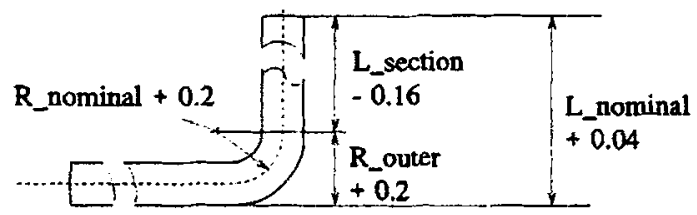

Figure 6: Error in length caused by a larger product radius

\section{THE CONVERSION OF TOLERANCES BETWEEN SUBSEQUENT PRODUCT STATES DURING PROC- ESS PLANNING}

4.1 The tolerance tree: a tool to represent relations in a set-up

The conversion of tolerances between subsequent product states during process planning is required for two reasons. Firstly, it can be checked whether the tolerance prescriptions are satisfied after each process step. Secondly, the values of the converted tolerances can be used to select one of the best promising alternative solutions. In this way, the propagation of constraints, imposed by tolerance prescriptions, can be kept under control. The concept of 'conversion of tolerances' is based on the generation of constraints for the product state prior to each bending operation. Figure 7 shows the relations between the faces in a bending set-up. At the top, the existing relation between the toleranced face 
and the reference face is shown. Both the position of the bending line with respect to the positioning face and the relation between the bending line and its adjacent faces, as shown at the bottom, can be calculated. The existing relation (tolerance) between the toleranced face and the reference face is replaced by two new relations, defined on the component before bending. In Figure 8 , two pairs of possible new relations are shown: pair 1) and pair 2). Which of these pairs is used, depends on the product in relation with the set-up configuration: each new pair is formed from paralle faces. In the case of holes, a face parallel to the centreline of the hole is selected.

\begin{tabular}{|c|c|c|c|c|}
\hline & $\begin{array}{l}\text { TOLERANCED } \\
\text { FACE }\end{array}$ & Tolerance & $\begin{array}{l}\text { REFERENCE } \\
\text { FACE }\end{array}$ & \\
\hline $\begin{array}{l}\text { POSITIONING } \\
\text { FACE }\end{array}$ & $\Delta \mathrm{P}$ & $\begin{array}{l}\text { BENDING } \\
\text { LINE }\end{array}$ & $\Delta L, \Delta \alpha$ & $\begin{array}{l}\text { ADJACENT } \\
\text { FACES }\end{array}$ \\
\hline
\end{tabular}

Figure 7: Existing and calculated relations in a bending set-up

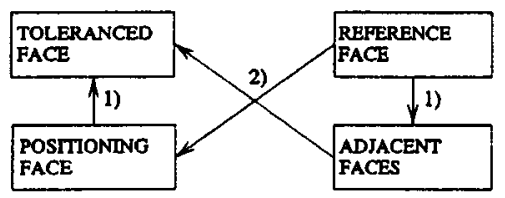

Figure 8: New relations, replacing the existing relation

The relations are stored in a so called tolerance tree (Figure 9). A tolerance tree belongs to one tolerance prescription, defined on the completed component. Each node in the tolerance tree represents a relation between a toleranced face and a reference face. The root node of a tolerance tree represents the initial tolerance prescription between two given faces. Therefore, in the root node, the initial tolerance value is stored in the 'value' box of the data format.

A node is evaluated when the accuracy in the relation between the considered faces is influenced by the bending operation in the set-up. During the evaluation of a node, two new nodes are added to the tree as child nodes of the considered node. A new node represents the relation between on the one hand the toleranced face or the reference face from its parent node, and on the other hand an adjacent face of the considered bend or the positioning face. The relations are indicated in Figure 8 . The initial value in the 'value' box of a new node is put to zero.

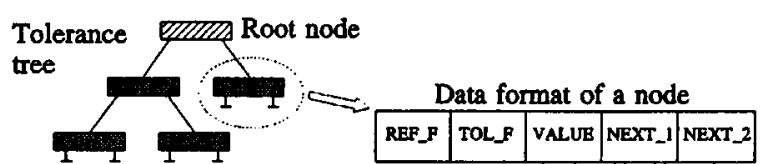

Figure 9: An example of a tolerance tree (left). The boxes in the data format of a node are shown to the right.

The positioning and bending errors are accounted for by the introduction of a penalty. The value of this penalty corresponds with the errors in the considered set-up. The value is subtracted from the value in the 'value' box, belonging to the parent node. The resulting tolerance value after a given evaluation step equals the original tolerance value minus the penalty values of the bends already dealt with. The considered set-up is not feasible when any tolerance value becomes negative.

When the accuracy of the relation represented by a node is not influenced by a bending operation, then the node has no child nodes, and is an end node. Otherwise, the node has two children. When at least one of the children is an end node, the conversion of tolerances is relatively straightforward, as shown in the example in Section 4.2.

\subsection{An example of the conversion of tolerances}

An example of a simple component, together with one of the alternative preceding set-ups, is shown in Figure 10. The faces are numbered I through VI. The tolerance prescription 'tol1' between the faces $I$ and $V$ is satisfied when the accuracy between the positioning face $V I$ and face $I$ is adequate. This is the case when the accuracy between the faces I and VI satisfies a new tolerance prescription 'tol1. 1 '. The same applies for the faces III and V where tol2 is trans- formed into tol2.1. The converted tolerances are calculated with the aid of tolerance trees.

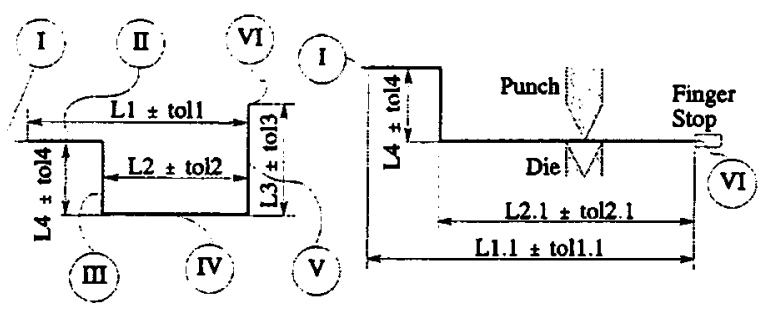

Figure 10: $\quad$ Cross section of a component (left) and a preceding set-up (right)

The initial tolerances, belonging to the respective pairs of faces in the completed component, are used as the initial tolerance values in the 'value' box of the root nodes. For tol1, the tolerance tree is shown in Figure 11. It consists of the root node and two nodes, added during the evaluation of the root node.

tree 1

\begin{tabular}{|c|c|c|c|c|}
\hline \begin{tabular}{|l|l|}
$\mathbf{V}$ & $\mathbf{I}$ \\
\end{tabular} & tol1-err1 & Ro & rode & \\
\hline II & 0 & \begin{tabular}{|l|l|}
$\mathrm{V}$ & $\mathbf{v}$ \\
\end{tabular} & 0 & $x \sqrt{x}$ \\
\hline
\end{tabular}

Figure 11: $\quad$ Tolerance tree, belonging to toll

According to the convention proposed in Section 2.1, the conventional tolerance 'toll' is replaced by a vectorial tolerance with face $V$ as the initial reference face. The initial toleranced face is face $I$. In the considered set-up, face VI serves as positioning face and face $V$ is an adjacent face of the bend. In this way, two new pairs of faces can be selected: $V \mid-I$ and $V-V$. The node with face pair $V-V$ is an end node, since it is obvious that the accuracy of face $V$ with respect to itself doesn't depend on any other bending operation. The crosses in the 'next' boxes of the data format indicate that the node is an end node. End nodes are added to the tree since the accuracy of the relation between the two involved faces can depend on other operations, e.g. nibbling or punching. This is the case for a side face and a hole, belonging to one main face.

Instead of a pair V.V, it is also possible to select a pair V-IV. This also results in an end node and in this case, the choice is arbitrary. In PART-S, the face which keeps the same orientation with respect to the other face during the bending operation is selected. This leads to more transparent calculations of the penalty values, especially for complex components.

The penalty 'err1' which is subtracted from the original tolerance value 'tol1' in the root node, consists of: (i) a positioning error $\Delta \mathrm{P}$, (ii) an error in length $\Delta \mathrm{L}$ and (iii) an angularity error $\Delta \alpha$ multiplied with L4.

The converted tolerance value 'tol1.1', replacing the initial value 'toll', can now be calculated as the sum of the values in the nodes of the tree:

tol1.1 $=\Sigma$ values $=$ tol1-err $1+0+0=$ tol1 $-\left(\Delta P+\Delta L+\Delta \alpha^{\star} L 4\right)$ The procedure for the calculation of tol2.1 is the same, but there are other faces involved. For tol3.1, the new nodes represent relations between the faces IV and IV and between the faces VI and VI respectively, as shown in Figure 12. Both new nodes in the tree are end nodes (indicated with the crosses). After the replacement of tol3 by tol3.1, the tree is completely evaluated. The considered set-up leads to an adequate accuracy of the component with respect to tol3 when the converted tolerance 'tol3.1' is non-negative. Tol3.1 is calculated from:

tol3.1 $=$ tol3-err3 $+0+0=$ tol3 $-(\Delta P+\Delta L) \geq 0$

tree3

$$
\text { IVIVI tol3-err3 }, \text {, Root node }
$$

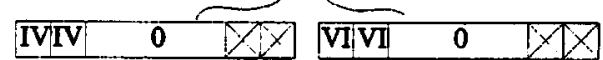

Figure 12: $\quad$ Tolerance tree with two end nodes, belonging to tol3 
In principle, the values of the converted tolerances are different for different set-ups. These values can be used to compare different set-ups with each other. The final selection of one of the set-ups is based on this evaluation and on collision and handling considerations.

In the example above, the values of the penalties and of the converted tolerances are calculated using arithmetic addition and subtraction. One reason to do this, and not for instance using the RSS (Root of the Sum of Squares) method, is that, in general, the errors don't compensate each other. Secondly, in small batch manufacturing, every component must be right, since rejects lead to serious additional costs. This also implies that adequate values for the errors must be used. For instance, the ' $3 \sigma$ ' boundaries obtained from statistical analysis can serve for this purpose. When the risk of rejects is acceptable, other methods can be used to calculate a converted tolerance value. Basically, this value is reduced stepwise when a set-up influences the accuracy of an involved relation (Figure 13).

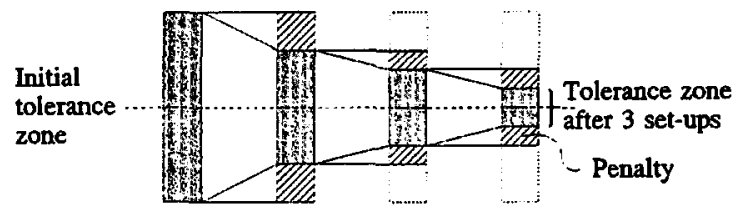

Figure 13: $\quad$ Stepwise reduction of the size of the tolerance zone

\subsection{Additional information in a tolerance tree}

Additional information can be attached to the nodes in the tolerance trees. In PART-S, the sweep length of the toleranced face is added. This additional information is required since the edges of a flattened bend are no longer present in the new CAD model, due to automatic 'clean up' of these edges after the flattening operation. In the example shown in Figure 10, flattening means that the faces IV and $V$ are one face after the flattening operation, and that the vertices belonging to the disappeared bend are lost.

It is convenient to use an indicator for an end node. When the indicator shows the value 'true', the node (including its children) is completely evaluated. When two nodes with the same parent are end nodes, then so is the parent node, and the end node indicator of the parent node is set to 'true'. Once a node has been identified as an end node, it remains an end node, no matter which bend is involved. In this way, it is possible to indicate which branches are already completely evaluated. With the use of end nodes, the required computing time for subsequent bending line evaluations reduces significantly. Completely evaluated tolerance trees (indicated by the end node indicator in the root node) are completely disregarded for the evaluation of alternative solutions. As mentioned before, when any converted tolerance value becomes smaller than zero, the corresponding set-up is not feasible, and thus removed from the set of alternatives. As a result, there is no risk that infeasible solutions are generated by disregarding already evaluated trees.

\section{CONCLUSIONS}

In the generation of bending sequences, the evaluation of alternative solutions is necessary in order to be able to select feasible solutions as regards the accuracy requirements. A methodology to deal with accuracy aspects is discussed in this paper. Since conventional tolerancing methods lead to ambiguous interpretation of the actual meaning of the tolerances, a convention for the vectorial interpretation of tolerances is required. The convention which is used in PART-S is discussed briefly. This convention forms a link between tolerancing practice and the theory of geometric tolerancing. In PART-S, tolerances are represented as relations between faces in the (B-rep) CAD model of a component.

With respect to bending operations, in PART-S, the tolerances are converted between subsequent product states. Thus, the propagation of the constraints, as imposed by the tolerances, is monitored systematically through the subsequent process planning steps. The converted tolerance values can be used in concurrency with handling and collision rules to select the most promising solution from a set of alternative set-ups. On the average, adequate set-up selection improves the accuracy of completed components without additional costs. For the future, more detailed estimation of bending errors seems to be a fruitful research topic.

\section{REFERENCES}

[1] Bjerke, Ø., 1978, Computer-Aided Tolerancing, Tapir Publ.

[2] Boerma, J.R., 1990. The Design of Fixtures for Prismatic Parts, PhD Thesis, University of Twente.

[3] Bourdet, P., Mathieu, L., (eds.), 1993, Proceedings of the 3rd CIRP Seminars on Computer Aided Tolerancing, Cachan.

[4] Clément, A., Desrochers, A., \& Rivière, A., 1991, Theory and Practice of 3-D Tolerancing for Assembly, CIRP International Working Seminar on Computer-Aided Tolerancing, Penn State University, 25-57.

[5] Clément, A., \& Rivière, A., 1993, Tolerancing versus nominal modeling in next generation CAD/CAM systems, 3rd CIRP Seminar on Computer Aided Tolerancing, 97-113.

[6] Elmaraghy, H.A., \& Elmaraghy, W.H., 1993, A System for Modeling Geometric Tolerances for Mechanical Design, 3rd CIRP Seminar on Computer Aided Tolerancing, 11-24.

[7] Hoffmann, M., Geißler, U., \& Geiger, M., 1992, Computer-aided generation of bending sequences for die-bending machines, $J$. of Mat Proc. Techn., 30:1-12.

[8] Houten. F.J.A.M. van, 1991, PART: a Computer Aided Process Planning System, PhD Thesis, University of Twente. [9] Jonkers, F.J.C.M., 1992, A Software Architecture for CAPP Systems, PhD Thesis, University of Twente.

[10] Klaassen, E., 1993, Bending sequence pre-determination and Collision checking for PART-S, MSc Thesis, University of Twente.

[11] Lee, J., \& Johnson, G.E., 1993, Optimal tolerance allotment using a genetic algorithm and truncated Monte Carlo simulation, Computer-Aided Design, 25/9:601-611.

[12] Lenderink, A., \& Kals, H.J.J., 1993, The Integration of Process Planning and Machine Loading in Small Batch Part Manufacturing, Robotics \& Comp. Int. Manuf., 10/1/2:89-98.

[13] Nassef, A., \& Elmaraghy, H.A., 1993, Allocation of Tolerance Types and Values using Genetic Algorithms, 3rd CIRP Seminar on Computer Aided Tolerancing, 147-156.

[14] Reissner, J., \& Ehrismann, R., 1990, Einsatz von Regelund Algorithmenbasierten Verfahren bei der Bestimmung von Biegefolgen, VDI Berichte 867:283-297.

[15] Requicha, A.A.G., 1983, Toward a Theory for Geometric Tolerancing, Int. J. of Robotics Res., 2/4:45-60.

[16] Sfantsikopoulos, M.M., 1993, Compatibility of Tolerancing, Int. Journ. of Adv. Manuf. Techn., 8:25-28.

[17] Stamp, R.J., \& Earl, C.F., 1992, Production of Sheet Metal Components by an Automatically Planned Robot Assisted Press Brake, Proc. of the Int. SheMet'92 Conf., Birmingham, 211-220.

[18] Streppel, A.H., Vin, L.J. de, Brinkman, J., \& Kals, H.J.J., 1993, Suitability of Sheet Bending Modelling Techniques in CAPP Applications, J. of Mat. Proc. Techn., 36:339-356.

[19] Vin, L.J. de, 1989, Sheet Metal Working Processes of Discrete Parts, MSc Thesis University of Twente, Enschede (in Dutch).

[20] Vin, L.J. de, Vries, J. de, Streppel, A.H., \& Kals, H.J.J., 1992, PART-S, a CAPP System for Small Batch Manufacturing of Sheet Metal Components, Proc. of the 24th CIRP Int. Sem. on Manuf. Sys., Copenhagen.

[21] Vin, L.J. de, Vries, J. de, Streppel, A.H., \& Kals, H.J.J., 1992. Computer Aided Process Planning for Small Batch Manufacturing of Sheet Metal Components, Proc. of the IMC-9 Conf., Dublin, 151-160.

[22] Vin, L.J. de, Streppel, A.H., Ittersum, E. van, \& Kals, H.J.J., 1993, Tolerancing and the Accuracy of Bending Operations in Sheet Metal Part Manufacturing, Proc. of the IMC-10 Conf., Galway, 423-436.

[23] Vries, J. de, Roosmalen, A.A.H.M. van, Streppel, A.H. \& Kals, H.J.J., 1993, Planning and Nesting in Small Batch Sheet Metal Manufacturing. Proc. of the 25th CIRP Int. Sem. on Manuf. Sys., Bled.

[24] Weill, R., 1988, Tolerancing for function, Ann. of the CIRP, 37/2:603-610.

[25] Zhang, X., \& Roy, U., 1993, Criteria for Establishing Datums in Manufactured Parts, J. of Manuf. Sys., 12/1:3650. 